\title{
Parry-Romberg syndrome: clinical, electrophysiological and neuroimaging correlations
}

\author{
Sławomir Budrewicz • Magdalena Koszewicz • \\ Ewa Koziorowska-Gawron • Pawel Szewczyk • \\ Ryszard Podemski · Krzysztof Słotwiński
}

Received: 4 May 2010/ Accepted: 23 August 2011/Published online: 10 September 2011

(C) The Author(s) 2011. This article is published with open access at Springerlink.com

\begin{abstract}
Parry-Romberg syndrome (PRS) is a rare disorder, described in the nineteenth century by Caleb Parry and Moritz Romberg, characterized by acquired and slowly progressive atrophy of one side of the face. The pathogenesis of PRS is still unclear. Immune-mediated processes are thought to be a basic factor in PRS etiology, but autonomic nervous system might also be impaired. A case of PRS in a 26-year-old woman with coexisting disturbances in the lower left limb is presented. The multimodal electrophysiological studies were done, including electroencephalography, visual, brain auditory, somatosensory and trigeminal somatosensory evoked potentials, blink reflex, standard neurographic and electromyographic examinations, quantitative sensory tests and autonomic tests. Neuroimaging studies consisted of brain MR, single voxel proton MR spectroscopy, diffusion tensor imaging with fiber tractography. Based on multimodal electrophysiological and neuroimaging studies, it was concluded that the impairment in PRS is multisystemic, i.e., motor, sensory, and autonomic. A cortical origin of the symptoms is possible.
\end{abstract}

Keywords Parry-Romberg syndrome . Electrophysiology $\cdot$ Neuroimaging

S. Budrewicz $(\varangle) \cdot$ M. Koszewicz · E. Koziorowska-Gawron · R. Podemski · K. Słotwiński

Department of Neurology, Wrocław Medical University, Borowska 213, 50-556 Wrocław, Poland

e-mail: s.budrewicz@wp.pl

P. Szewczyk

Department of General Radiology, Interventional Radiology and Neuroradiology, Wrocław Medical University, Borowska 213, 50-556 Wrocław, Poland

\section{Introduction}

Parry-Romberg syndrome (PRS), also called progressive hemifacial atrophy, was described in 1825 by Caleb Parry and then elaborated upon by Moritz Romberg in 1846 [1]. PRS is characterized by acquired, slowly progressive, selflimited, unilateral atrophy of the face which primarily affects subcutaneous tissue, fat, and connective tissue, then the skin, muscles, and bones [1-4]. The pathogenesis of PRS is still uncertain and seems to be heterogeneous [1-5]. Immune-mediated processes are primarily considered. Wartenberg [6] concluded that disturbed central regulation leads to hyperactivity of the sympathetic nervous system, which controls the trophism of fat and subcutaneous tissue. Electrophysiological studies of PRS patients usually did not reveal characteristic changes [1, 2, 7]. Brain MR abnormalities are usually ipsilateral to the affected part of the face, but contralateral or bilateral changes can be seen as well $[8,9]$. Considering this, we conducted sophisticated diagnostic procedures.

\section{Case}

We describe a 26-year-old woman (M.S.) who developed progressive right facial hemiatrophy at the age of 5 which became stable 5 years later (Fig. 1). She was admitted to the Department of Neurology when she was 26 years old because of progressive weakness of the left lower limb lasting 1 year. Clinical examination revealed atrophy of the facial and lingual subcutaneous tissue and muscles on the right side, a definite vertical line reaching the eyebrows on the forehead, hyperpigmentation of the right orbicular area, and pterygium of the right eye. Slight monoparesis of the left lower limb with exaggerated tendon reflexes and 


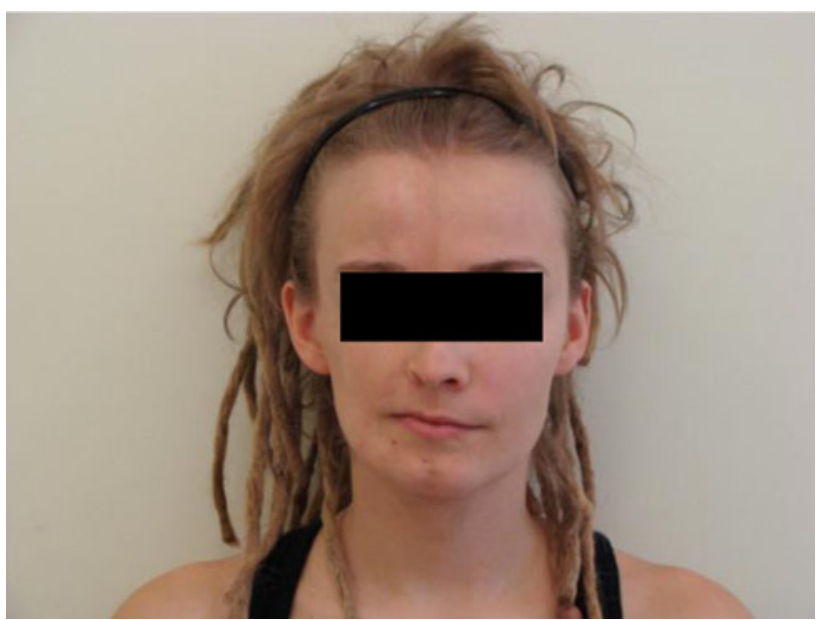

Fig. 1 M.S., 26 years old. Progressive facial hemiatrophy (PFH) on the right side

Babinski's sign, vascular symptoms such as cooling at the left shank and foot, and lividity of the toes of the left foot were also found. The patient was fully mobile. Neuropsychological examination showed no cognitive impairment. The antibody profile revealed the presence of antinuclear antibodies (ANAs-1:100). The level of protein in the cerebrospinal fluid was slightly elevated $(53 \mathrm{mg} \%)$. No oligoclonal IgG bands were found. The Qalb and Eindex IgG ratios were within normal limits. Other blood parameters (morphology, biochemistry, CRP, ENA, LA, etc.) were also within normal limits. Ultrasonographic Dopplerduplex examination of the arteries and veins of the lower limbs did not display any pathological changes except for a narrow vascular lumen of distal arteries, suggesting vascular spasm. Electroencephalography (EEG) was normal. In the blink reflex (BR) study, the R2 and R2' latencies were prolonged $\left(\mathrm{R} 2=39.7 \mathrm{~ms}, \mathrm{R} 2^{\prime}=39.7 \mathrm{~ms}\right)$ after stimulation on the left side, but were within the normal limit $\left(\mathrm{R} 2=31 \mathrm{~ms}, \mathrm{R} 2^{\prime}=31 \mathrm{~ms}\right)$ after stimulation of the right side. Recording electrodes were placed on the both orbicularis oculi muscles (active) and on the nose (reference), and stimulating electrodes were placed over the supraorbital foramina. The amplitudes of brain auditory evoked potentials (BAEPs) were lower on the right side, without any differences in latencies. Left trigeminal somatosensory evoked potentials (TSEP) had mildly longer latencies than those stimulated and recorded on the opposite side. No abnormalities were found in visual evoked potentials (VEPs) and somatosensory evoked potentials (SSEPs) from the upper and lower extremities (stimulation of median and tibial nerves bilaterally). We used standard techniques and parameters of stimulation for all evoked potentials. Electromyographic examination of the left orbicular muscle of eye and neurography of both facial nerves were within the normal limits.
Electroneurography and EMG of the four extremities were also correct, except for a low amplitude of motor responses in both peroneal nerves (motor conduction velocities were normal). Neurosensory analysis (quantitative sensory tests) discovered abnormal temperature and pain thresholds (cold pain difference between the sides was $4.1^{\circ} \mathrm{C}$, heat pain $4.4^{\circ} \mathrm{C}$ ) in the lower left limb. The cold sensation threshold was mainly elevated and the difference between the sides was $8.8^{\circ} \mathrm{C}$, while the warm sensation difference was only $2.6^{\circ} \mathrm{C}$ (Table 1 ). There was no sympathetic skin response (SSR) in the left foot. SSR in both hands and the right foot were present with the correct latencies. Heart rate variability (HRV) analysis did not show any pathological findings. Brain MR showed thinning and atrophy of the upper frontal gyrus, the medial part of precentral gyrus and paracentral lobule of the right frontal lobe on T2-weighted images and discrete hiperintensity of the white matter in the upper part of the right frontal lobe (corona radiata) on T2-weighted and FLAIR images (Fig. 2a, b). Single voxel proton MR spectroscopy $(1 \mathrm{H}-$ MRS) displayed decreased level of $\mathrm{N}$-acetylaspartate (NAA) and increased level of choline (Cho) and mioinositol (mI) in the upper part of the right frontal lobe (Fig. 3a, b). Diffusion tensor imaging (DTI) displayed significantly decreased fractional anisotropy (FA) parameter in the white matter of right upper frontal gyrus (61\% of FA in the left upper frontal gyrus) (Fig. 4a) and in the upper part of the right corona radiata. Fiber tractography derived from DTI showed the reduction amount of fibers and involvement of the right pyramidal tract (Fig. 4b). Lumbar-sacral part of vertebral column MR revealed small central intervertebral disc protrusions on level L4/L5 and L5/S1.

\section{Discussion}

The pathogenesis of PRS is still unclear. Immune-mediated processes are thought to be a basic factor in the etiology of PRS. According to the literature, the autonomic nervous

Table 1 Quantitative somatosensory test: comparison of the left and right side

\begin{tabular}{|c|c|c|c|c|}
\hline & $\mathrm{CS}\left({ }^{\circ} \mathrm{C}\right)$ & WS $\left({ }^{\circ} \mathrm{C}\right)$ & $\mathrm{CP}\left({ }^{\circ} \mathrm{C}\right)$ & $\overline{\mathrm{HP}\left({ }^{\circ} \mathrm{C}\right)}$ \\
\hline Right hand (C6) & 30.8 & 33.7 & 30.9 & 33.6 \\
\hline Left hand (C6) & 29.9 & 33.6 & 30.9 & 33.7 \\
\hline Difference $(\Delta)$ & 0.9 & 0.1 & 0 & 0.1 \\
\hline Right foot (S1) & 29.5 & 38.5 & 30.2 & 40.0 \\
\hline Left foot (S1) & 20.7 & 41.1 & 26.1 & 44.5 \\
\hline Difference $(\Delta)$ & 8.9 & 2.6 & 4.1 & 4.5 \\
\hline
\end{tabular}

$C S$ cold sensation threshold, WS warm sensation threshold, $C P$ cold pain threshold, $H P$ heat pain threshold 


\section{(a)}

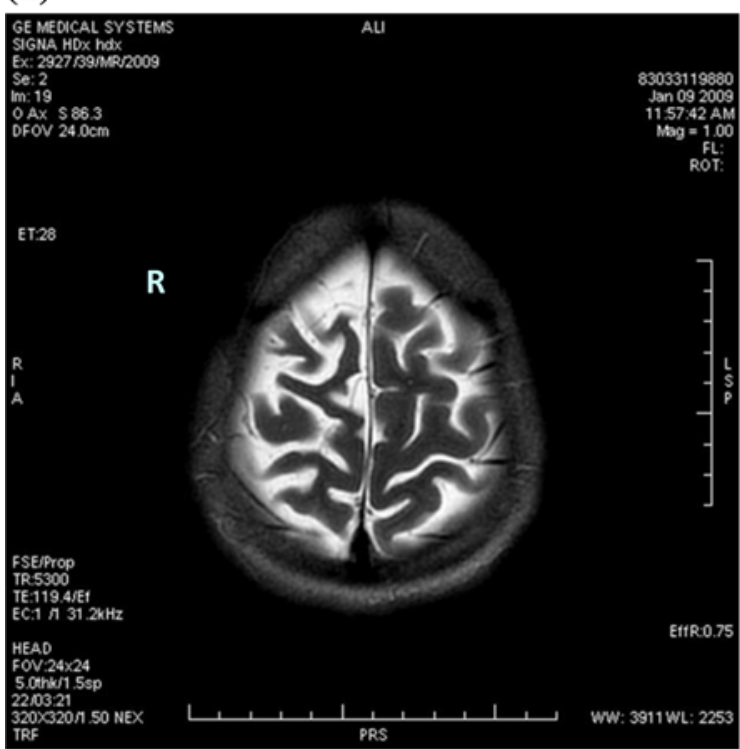

Fig. 2 MRI, axial FSE T2-weighted images. a The atrophy of the upper frontal gyrus, paracentral lobule and the medial part of precentral gyrus in the right $(R)$ frontal lobe. b Relative sparing of the

\section{(b)}

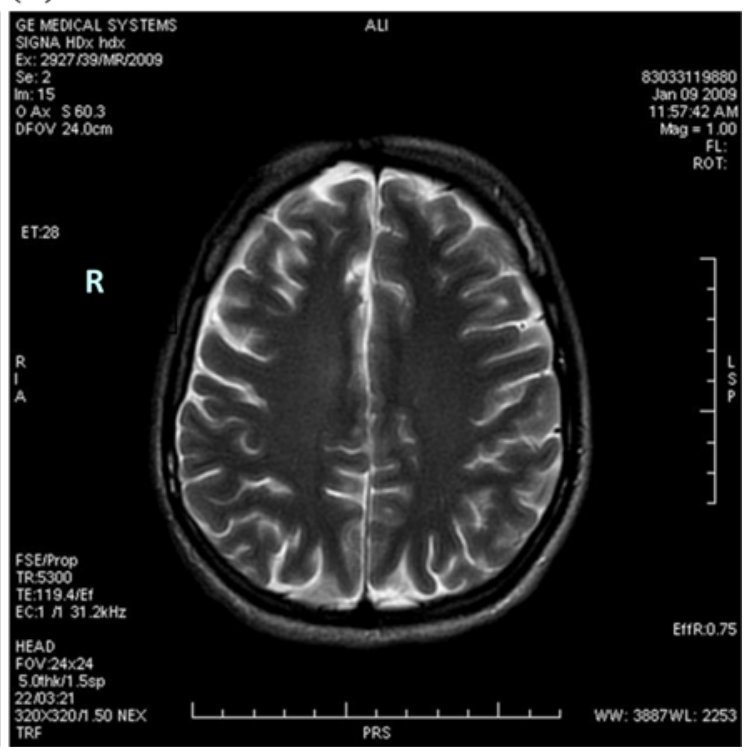

lateral part of precentral gyrus and discrete hiperintensity of the white matter in the upper part of the right $(R)$ frontal lobe

(b)

(a)

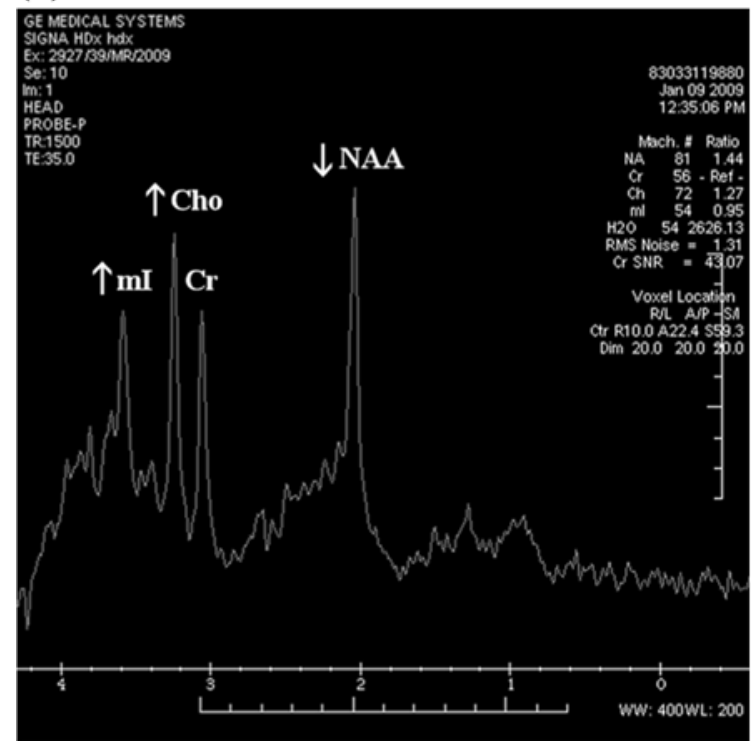

Fig. $3{ }^{1} \mathrm{H}-\mathrm{MR}$ spectroscopy, single voxel method. a Spectrum obtained from a voxel located in the subcortical white matter of involved right frontal lobe revealed decreased level of $\mathrm{N}$ -

system (eventually the superior cervical ganglion) might also be impaired. In PRS patients, changes were seen using different diagnostic procedures (e.g. neuroimaging, electrophysilogical) [1, 7-11]. We emphasize the diversity of clinical features as well as diagnostic results seen in our patient with confirmed PRS. The prolongation of the R2 and R2' latencies after left-side stimulation is partly

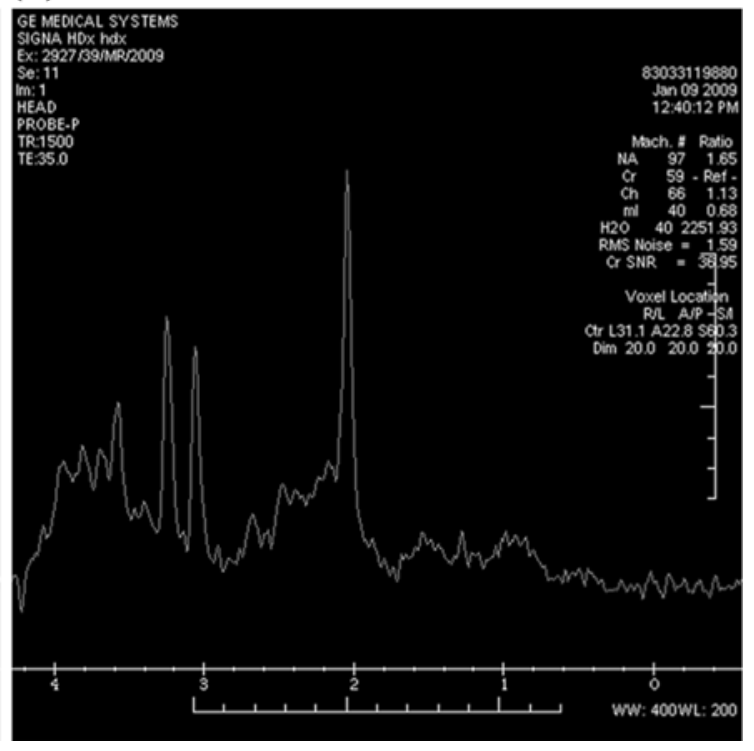

acetylaspartate (NAA) and increased level of choline (Cho) and mioinositol $(\mathrm{mI})$ in comparison to control voxel (intrinsic control) located in the corresponding area in left frontal lobe (b)

compatible with previous reports. Lonchampt et al. [10] found a lack of R2 and R2' components after stimulation of the affected side in a patient with PRS and a lack of R2' components in the response of stimulation of the opposite side. According to Kimura [12], R2 components could be significantly affected by a contralateral hemispheric lesion. Our findings are probably connected with the damage to 
(a)

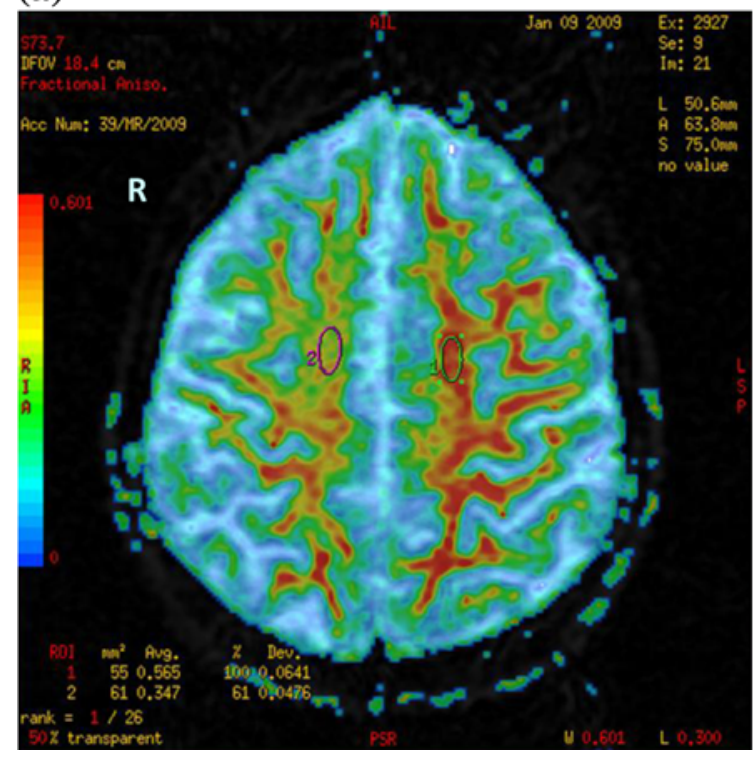

Fig. 4 MR Diffusion tensor imaging (DTI). a Fractional anisotropy parametric map shows significantly decreased fractional anisotropy (FA) value $(0.476)$ of the white matter in the right $(R)$ upper frontal

the right hemisphere, but a brain stem lesion could not be completely excluded (lateral medulla, spinal nucleus). The changes could be due to a left-sided trigeminal lesion (particularly as both R2 and R2' were prolonged following left sided stimulation). These observations were supported by the neuroimaging studies performed in our patient. $1 \mathrm{H}-$ MRS abnormalities might imply damage of the white matter secondary to changes in the cortex of the right frontal or primary damage of axons and gliosis. DTI and fiber tractography derived from DTI indicated moderate damage of white matter fiber tracts in the right upper frontal lobe, the upper part of the right corona radiata and pyramidal tract. The atrophy of the right upper frontal gyrus and the precentral gyrus was agreeable with clinical picture, and in accordance with brain somatic field arrangement $[13,14]$. They are similar to the Moon et al. results [15].

We did not find any changes in standard neurography and electromyography. Axonal impairment of both peroneal nerves is thought to be a congenital anomaly. We did not find any other explanation of the anomaly, although there were no accessory peroneal nerves. In the neurosensory analysis we found significant differences in the temperature and pain thresholds between the lower limbs. They were higher on the left side, much more so when the cold sensation was considered. These findings might indicate a small-fiber lesion (Adelta) or central pathway impairment $[7,12,16-18]$. They were connected with the absence of an SSR, which was also restricted to the left lower limb. There was no linear relationship between SSR amplitude and (b)

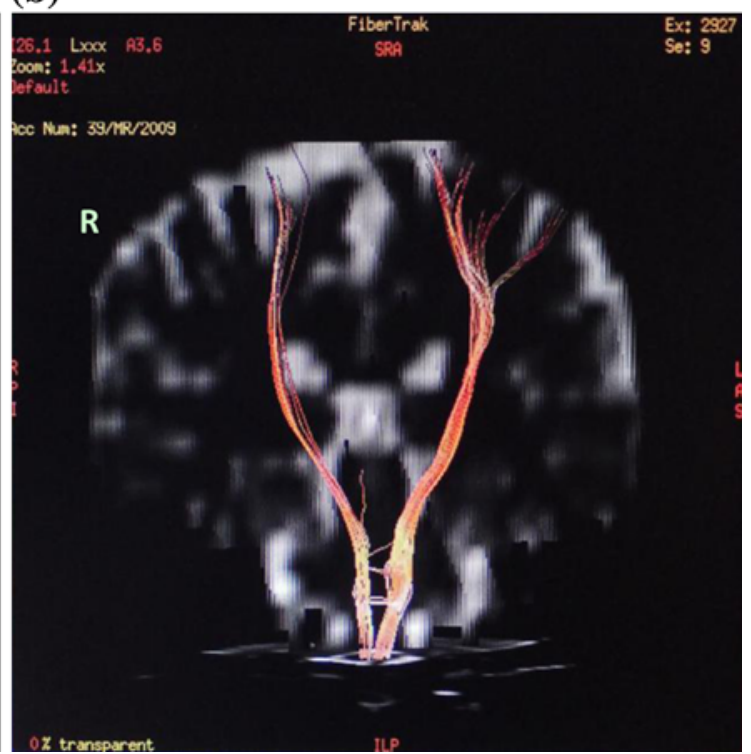

gyrus in comparison to corresponding area in the left upper frontal gyrus (0.641). b Fiber tractography shows involvement of the right $(R)$ pyramidal tract (reduction the amount of fibers)

sweat gland function [19]. Therefore, over-reactivity of the sympathetic nervous system could not be excluded by the lack of an SSR. The reflex arch of the SSR is polysynaptic and consists of the reticular formation, hypothalamus, limbic system, and contralateral cerebral cortex. Lesion of ventromedial prefrontal cortex is consistent with a lack of SSR. In the nervous system there are many common points of the autonomic and sensory/nociceptive pathways (posterior horn of the spinal cord, reticular formation, hypothalamus, frontal cortex). According to our findings in the clinical examination, MRI, and electrophysiological procedures, changes in the lower left limb of our patient seem to result from a lesion of the central nervous system. We are not able to explain this because there were no changes in the upper left limb. The very late onset of the weakness (about 20 years after the first symptoms) could be connected with the enhancement of the immune-mediated processes (currently ANAs and CSF protein levels elevation). We cannot completely exclude a peripheral lesion of the lower left limb. Although the lumbar MRI did not reveal any important changes, sympathetic impairment might be located in the L1-L2 segments of the spinal cord, in the ganglia (lumbar and sacral part of sympathetic trunk), or in postganglionic fibers. We indicated that in this patient, sympathetic disturbances were not limited to the face region, but were also present in the opposite lower limb, without any changes in the upper limb. The patient also presented pyramidal signs located in the left lower limb as well as sensory deficit at the same place. The definite neuroimaging changes in the right frontal lobe very 
closely corresponded with clinical motor symptoms. We emphasize the primary, multisystemic changes in the nervous system in the pathogenesis of PRS. Based on electrophysiological and neuroimaging procedures, we conclude that the PRS signs might be of cortical origin.

Open Access This article is distributed under the terms of the Creative Commons Attribution Noncommercial License which permits any noncommercial use, distribution, and reproduction in any medium, provided the original author(s) and source are credited.

\section{References}

1. Paprocka J, Jamroz E, Adamek D, Marszał E, Mandera M (2006) Difficulties in differentiation of Parry-Romberg syndrome, unilateral facial sclerodermia, and Rasmussen syndrome. Childs Nerv Syst 22:409-415

2. Tollefson MM, Witman PM (2007) En coup de sabre morphea and Parry-Romberg syndrome: A retrospective review of 54 patients. J Am Acad Dermatol 65:257-263

3. Roller E, Reifenberger J, Homey B, Bruch-Gerharz D (2006) Hemiatrophia faciei progressiva (Parry-Romberg-syndrome). Hautarzt 10:905-906

4. Drummond PD, Hassard S, Finch PM (2006) Trigeminal neuralgia, migraine and sympathetic hyperactivity in a patient with Parry-Romberg syndrome. Cephalalgia 26:1146-1149

5. Błaszczyk M, Królicki L, Krasu M, Glinska O, Jabłońska S (2003) Progressive facial hemiatrophy: central nervous system involvement and relationship with scleroderma en cup de saber. J Rheumatol 30:1997-2004

6. Wartenberg R (1945) Progressive facial hemiatrophy. Arch Neurol Psychiat 54:75-96

7. Zambelis T, Tsivgoulis G, Kokotis P, Spengos K, Karandreas N (2008) Electrophysiological findings in a case of congenital lower limb hypoplasia. Neurol Sci 29:177-179
8. Cory RC, Clayman DA, Faillace WJ (1997) Clinical and radiologic findings in progressive facial hemiatrophy. Am J Neuroradiol 18:751-754

9. Moko SB, Mistry Y, Blandin de Chalain TM (2003) ParryRomberg syndrome: intracranial MRI appearances. J Craniomaxillofac Surg 31:321-324

10. Lonchampt P, Emile J, Pelier-Cady MC, Cadou B, Barthelaix A (1995) Central sympathetic dysregulation and immunological abnormalities in a case of progressive facial hemiatrophy. Clin Auton Res 5:199-204

11. Resende LA, Dal P, Alves A (1991) Experimental study of progressive facial hemiatrophy: effect of cervical sympathectomy in animal. Rev Neurol 147:609-611

12. Kimura J, Wilkinson JT, Damasio H, Adams HR, Shivapour E, Yamada T (1985) Blink reflex in patients with hemispheric cerebrovascular accident (CVA), Blink reflex in CVA. J Neurol Sci $67: 15-28$

13. Taylor HM, Robinson R, Cox T (1997) Progressive facial hemiatrophy: MRI appearance. Dev Med Child Neurol 39(7):484-486

14. Okamura A, Ikuta T, Tsuji T, Kato T, Fukatsu H, Naganawa S, Kato K, Watanabe K (2006) Perry-Romberg syndrome with a clinically silent white matter lesion. Am J Neuroradiol 27:1729_ 1731

15. Moon WJ, Kim HJ, Roh HG, Oh J, Han SH (2008) Diffusion tensor imaging and fiber tractography in Parry-Romberg Syndrome. AJNR 29:714-715

16. Chéliout-Héraut F, Zrek N, Khemliche H, Varnet O, Seret-Begue D, Martinez M, Nizou R, Bour F (2005) Exploration of small fibres for testing diabetic neuropathies. Joint Bone Spine 72:412415

17. Yarnitsky D, Ochoa JL (1990) Studies of heat pain sensation in man: perception thresholds, rate of stimulus rise and reaction time. Pain 40:85-91

18. Malndrini A, Dotti MT, Federico A (1997) Selective ipsilateral neuromuscular involvement in a case of facial and somatic hemiatrophy. Muscle Nerve 20:890-892

19. Low PA (1997) Clinical autonomic disorders. Lippincott-Raven, Philadelphia 